MMW Kommentar

Das Urteil steht im Widerspruch zu dem Inhalt eines Schreibens vom 4.4.2012, in dem das Bundesgesundheitsministerium (BMG) ausführlich darlegt, warum die Beratungsregelung auch auf Verfahren Anwendung findet, die Verordnungen vor 2012 betreffen. Damit wird das Thema zu einer Art "Hängepartie", denn gegen die Anwendung der Beratungsregelung auf Prüfverfahren bezüglich der Verordnungsjahre bis 2011 vertritt mittlerweile auch das Bundessozialgericht (BSG) die Auffassung, dass auf Wirtschaftlichkeitsprüfungen diejenigen materiellen Regelungen anzuwenden wären, die im Prüfquartal galten. Etwas anderes gelte nur für Vorschriften über das formelle Verfahren (AZ.: B 6 KA 34/07 R). Eine Rückwirkung der Beratungsregelung zu Lasten der Krankenkassen sei unzulässig. Hier ist allerdings nicht das letzte Wort gesprochen, denn es geht hier um die Deutung des Begriffs „Rückwirkung". Die Neuregelung zur Beratungspflicht wirkt nämlich nicht zurück auf Verordnungen vergangener Jahre, sondern wirkt für jetzt durchgeführte Prüfverfahren. Geregelt wird nichts Vergangenes, sondern das, was die Prüfgremien heute zu tun haben. Das BSG-Urteil beschreibt deshalb allenfalls die Rechtslage, aus der sich ergibt, dass formelle Regelungen zu Zuständigkeit, Verfahren und Form so anzuwenden sind, wie sie zum Entscheidungszeitpunkt gelten.

Sowohl Prüfungsstelle als auch Beschwerdeausschuss müssen deshalb im Jahr 2012 die Beratungsregelung anwenden, egal welches Verordnungsjahr betroffen ist. Lediglich, wenn - wie im Fall des Düsseldorfer Urteils - bereits ein Richtgrößenregress vor 2012 festgesetzt wurde, gilt der allgemeine Grundsatz, dass die Gerichte dasjenige Recht anwenden müssen, das zum Zeitpunkt der überprüften Behördenentscheidung bestand.

\title{
Welches Ultraschallgerät kann noch verwendet werden?
}

— Die neue Ultraschallvereinbarung sorgt immer noch für Verwirrung. So ist es nicht möglich, pauschal zu sagen, welche Ultraschallsysteme die Anforderungen der neuen Ultraschall-Vereinbarung, die seit 1. April 2009 gilt, erfüllen. In dieser neuen Ultraschall-Vereinbarung wurden u. a. verschiedene technische Anforderungen geändert, daher kann nur ein Techniker beurteilen, ob ein Gerät diese Anforderungen erfüllt oder nicht. Dazu gibt es ein Formular, das vom jeweiligen Gerätehersteller bzw. Lieferanten auszufüllen ist.

Mit dieser sogenannten Gewährleistungserklärung des Herstellers und der Vorlage von mindestens einer aktuellen Bilddokumentation pro Schallkopf wird gegenüber der zuständigen Kassenärztlichen Vereinigung (KV) nachgewiesen, dass ein Gerät der neuen Ultraschall-Vereinbarung entspricht. Die Prüfung der vorgelegten Bilddokumentationen erfolgt durch die jeweilige Sonografie-Kommission. Dieses Procedere ist die so genannte Abnahmeprüfung gemäß §9 der Ultraschall-Vereinbarung, die für jedes Ultraschallsystem durchgeführt werden muss.

\section{MMW Kommentar}

Für Ultraschallsysteme, die schon vor dem 1. April 2009 im Einsatz waren, gilt gemäß $\$ 16$ der Ultraschall-Vereinbarung die Übergangsregelung bis 31. März 2013, d. h. bis dahin muss auch für diese so genannten Bestandsgeräte eine Abnahmeprüfung durchgeführt werden, um nachzuweisen, dass sie den gültigen Bestimmungen entsprechen.

\section{Achtung:}

\section{Hier muss der Dummy durch eine Anzeige ersetzt werden !!}

